\title{
NRC E INRA PARA RACIONES DE CABALLOS DE OCIO BASA- DAS EN FORRAJES SECOS Y CONCENTRADOS GRANULADOS
}

\author{
NRC VS. INRA TO FORMULATE RATIONS BASED ON DRY ROUGHAGES AND \\ PELLETED CONCENTRATES FOR LEISURE HORSES
}

\author{
Martínez Marín, A.L. ${ }^{1}$ \\ 1Departamento de Producción Animal. Universidad de Córdoba. Campus de Rabanales. 14071 Córdoba.
} España.pa1martm@uco.es

\author{
Palabras clave adicionales \\ Equinos. Nutrición. Formulación.
}

\section{RESUMEN}

El objetivo del presente trabajo fue estudiar la influencia del sistema de valoración de alimentos y de necesidades nutritivas en el coste y características nutricionales de raciones para caballos estabulados, sin acceso a pastos, en diferentes estados fisiológicos (gestación, lactación, crecimiento y ejercicio ligero). Se optimizaron 24 raciones mediante programación lineal a mínimo coste con uno de tres forrajes (heno de alfalfa, paja de cereales o una combinación de ambos al $50 \%$ ) y catorce materias primas comúnmente usadas en España para la incorporación a concentrados granulados para caballos. El coste asignado a las materias primas correspondió a su precio de mercado durante los meses previos a la realización del trabajo. Utilizando una composición química de referencia se calculó el valor energético y proteico de las materias primas de acuerdo a las publicaciones de National Research Council, NRC (1989) e Institut National de la Recherche Agronomique, INRA (1990). Las necesidades de energía, proteína y lisina de los caballos en los cuatro estados fisiológicos considerados también se tomaron de ambas publicaciones. El resto de necesidades nutritivas y las restricciones aplicadas tanto a los nutrientes como a los ingredientes se igualó para los dos sistemas siguiendo referencias bibliográficas. El consumo diario posible de materia seca se acotó de acuerdo al estado fisiológico según valores coherentes para ambos sistemas. Se obtuvieron 23 soluciones válidas, encontrándose que las raciones más económicas fueron las basadas en paja de cereales como único forraje. Hubo diferencias, variables según

Recibido: 30-9-07. Aceptado: 5-11-07.

\author{
AdDitiOnAL KEYWORDS \\ Equines. Nutrition. Formulation.
}

el estado, en el consumo diario calculado de materia seca total, de forraje y de concentrado, y en el coste entre las raciones de ambos sistemas. El contenido de energía y almidón también fue diferente entre los sistemas sobre todo en las raciones para yeguas lactantes y en las de paja de cereales. Con independencia del sistema, el exceso de proteína sobre las necesidades mínimas fue mayor en todas las raciones con heno de alfalfa.

\section{SUMMARY}

This work was aimed to find out the effect of the feeding system on the cost and nutritional characteristics of the diets used for non-grazing horses in different physiological or working conditions (pregnancy, lactation, growth and light exercise). Working with a minimum cost, linear programming software, limits of ingredients and nutrients for twenty four diets were set using, a) one of three fibrous feedstuffs (alfalfa hay, cereal straw and a 50:50 combination of them) as a fixed component, and fourteen other variable ingredients commonly used in Spain to formulate pelleted, concentrate compound feedstuffs for horses; b) energy and protein values of every ingredient calculated according to NRC (1989) and INRA (1990) horse feeding systems on the basis of a chemical composition of reference for each feedstuff; c) energy, protein and lysine requirements of the animals, also taken from both feeding systems, and d) limits applied to other nutrients and to the feedstuffs, based in published 
literature, which were the same whatever the system used to formulate the diets. A limit of voluntary intake was set for each type of animal according to values consistent with both feeding systems. Twenty three valid solutions were found, the lowest cost of them being those with cereal straw as the only permanent roughage. Differences between systems, of variable size according to physiological conditions, were found for the calculated intake of roughage, concentrate and total daily ration, and for the cost of the daily ration. Energy and starch contents of the rations were also different between systems mainly of those of lactating mares and of those with cereal straw. The excess of dietary protein over the protein requirements was always higher in the rations with alfalfa hay as the only roughage, independently of the feeding system used to formulate the diets.

\section{INTRODUCCIÓN}

En España, el elevado número de caballos alojados en establos particulares y en centros deportivos periurbanos donde el espacio es limitado y no hay acceso a pastos crea la necesidad de diseñar raciones para estos animales de fácil reparto en los pesebres, capaces de satisfacer económicamente las necesidades nutritivas diarias y lo suficientemente seguras para prevenir la ocurrencia de trastornos digestivos. La práctica habitual es el reparto dos veces al día de una ración constituida por un forraje y un concentrado en cantidad variable dependiendo del estado fisiológico. Para diseñar el tipo de raciones mencionadas, el nutricionista debe construir o disponer de una matriz de formulación que contenga los valores de composición química de las materias primas disponibles (preferiblemente datos propios obtenidos por análisis químico o con tecnología NIR o, si ello no es posible, derivados de alguna de las tablas publicadas de composición de alimentos como las de la Fundación Española para el Desarrollo de la Nutrición Animal (FEDNA, 2003). Si dispone de valores analíticos propios de las materias primas, el nutricionista podrá estimar de forma más precisa su valor nutritivo según alguna de las ecuaciones de predicción publicadas para alimentos de caballos. Por último, con la matriz construida y teniendo en cuenta los precios de las materias primas, diseñará raciones que, en base a un criterio de economía, cubran las necesidades nutritivas diarias de los caballos en función de su estado fisiológico y de su capacidad de consumo. Las necesidades nutritivas de los caballos serán estimadas a su vez según alguno de los diferentes sistemas de alimentación desarrollados por organismos de investigación (National Research Council-NRC-, Institut National de la Recherche Agronomique -INRA-, etc.). El coste final de la fórmula diseñada debería depender no sólo de los precios de las materias primas sino también de la elección del sistema de valoración nutricional de los alimentos y de cálculo de las necesidades nutritivas de los animales. Por ello, el objetivo del presente estudio fue comparar el coste y la composición de las raciones para caballos en diferentes estados fisiológicos según los dos sistemas de valoración de alimentos y de cálculo de necesidades comúnmente empleados en España basados en las publicaciones de NRC de 1989 Nutrient Requirements of Horses (NRC89) y en la de INRA de 1990 L'Alimentation des Chevaux(INRA90).

\section{MATERIAL Y MÉTODOS}

\section{ANImALES}

Para el estudio comparativo se consideraron 4 estados fisiológicos: yeguas en el undécimo mes de gestación, yeguas con una producción lechera de $15 \mathrm{~kg} / \mathrm{d}$ (ambos grupos en buen estado de carnes), potros de 12 meses de edad creciendo $0,65 \mathrm{~kg} / \mathrm{d}$, y caballos sometidos a 1-2 horas de ejercicio ligero. El animal modelo fue un caballo con un peso vivo (PV) adulto de $500 \mathrm{~kg}$.

\section{NeCEsIDADESNUTRITIVAS}

Las necesidades nutritivas fueron tomadas de las tablas correspondientes de las 


\section{SISTEMAS NRC E INRA Y RACIONES A MÍNIMO COSTE PARA CABALLOS DE OCIO}

publicaciones de NRC89 e INRA90, previa comprobación de que las descripciones de los estados de los animales eran equivalentes (Martin-Rosset, 2001). En cuanto a la energía y la proteína, brevemente, NRC89 expone las necesidades en energía digestible (ED) y proteína bruta (PB) en tanto que INRA90 utiliza la energía neta (EN), en relación a la cebada de referencia (Unidades Forrajeras para Caballos-UFC), y la proteína digestible corregida por su aporte de aminoácidos (Materias Nitrogenadas Digestibles para Caballos -MNDC). Ninguno de ambos sistemas especifica necesidades de fibra bruta y almidón. NRC89 indica unas necesidades mínimas de lisina para cada estado fisiológico pero INRA90 únicamente precisa requerimientos de lisina para los potros. Para simplificar los cálculos, las necesidades de minerales se igualaron en ambos sistemas y, tras contrastar diversas fuentes (Wolter, 1977; NRC, 1989; INRA, 1990; McCutcheon, 2001; Coenen, 2001), los valores de necesidades minerales indicados en la publicación de Wolter (Wolter, 1977) se consideraron coherentes para los dos sistemas, salvo en el caso de los potros en que se utilizó la media de los valores propuestos por NRC89 e INRA90. La relación calcio/fósforo se consideró aceptable entre $1,2 / 1$ y $3 / 1$ en los potros y entre $1,2 / 1$ y 6/1 en los adultos (Wolter, 1977; NRC, 1989; INRA, 1990; Lewis, 1991).

\section{Alimentos}

Para calcular la concentración energética de la ración se supuso un consumo de materia seca total coherente con los valores propuestos por NRC89 e INRA90 comprendido entre el 1,5 y el 2\% PV en las yeguas gestantes y los caballos y del 2 al 2,5\% PV en las yeguas lactantes y los potros. La separación de los cuatro estados fisiológicos en dos grupos de consumo se justificó por la similitud de sus requerimientos de energía expresados en función del peso vivo metabólico $\left(\mathrm{PV}^{0,75}\right)$. La ración se consideró compuesta por forraje y un concentra- do granulado en el que estarían incluidos el resto de materias primas, los minerales y la premezcla de vitaminas y oligoelementos. Los forrajes -heno de alfalfa, paja de cereales y una combinación de ambos al 50\%- se eligieron por su disponibilidad y uso común en nuestra área geográfica (Rivera, comunicación personal). Las materias primas escogidas para la formulación del concentrado son habitualmente empleadas en España para la fabricación de piensos complementarios granulados para caballos. Para garantizar la seguridad digestiva de las raciones se fijó un aporte mínimo de forraje igual a 1 kg por cada $100 \mathrm{~kg}$ PV (Tisserand, 1979; Meyer, 1987; NRC, 1989; Lewis, 1991; Coenen, 2001). Por el mismo motivo, se supuso que la ración se repartiría en dos veces por día (Lewis, 1991) siendo aportado el forraje antes que el concentrado (Zeyner et $a l ., 2004)$. La composición química de referencia de las materias primas utilizadas en la formulación (tabla I) se tomó de las tablas de

Tabla I. Valores nutricionales calculados (sobre materia seca)*. (Calculated nutritional content of the feedstuffs, DM basis).

\begin{tabular}{lccc}
\hline & EDNRC & & \\
& Mcal/kg & UFC/kg & MNDC/kg \\
\hline Heno de alfalfa & 2,22 & 0,54 & 100 \\
Paja de cereales & 1,66 & 0,30 & 2 \\
Avena & 3,11 & 1,01 & 95 \\
Cebada 6 carreras & 3,66 & 1,12 & 102 \\
Maíz nacional & 3,88 & 1,33 & 71 \\
Salvado de trigo & 3,33 & 0,91 & 133 \\
Melaza de remolacha & 3,37 & 1,18 & 101 \\
Harina de girasol 30 & 2,22 & 0,67 & 262 \\
Harina de soja 44 & 3,63 & 0,91 & 443 \\
Garrofa & 1,83 & 0,64 & 16 \\
Alfalfa granulada & 2,41 & 0,61 & 110 \\
Pulpa de remolacha & 2,67 & 0,84 & 50 \\
Aceite de soja & 9,00 & 2,96 & 0 \\
\hline
\end{tabular}

*A partir de la composición química (FEDNA, 2003). Abreviaturas: $\mathrm{ED}=$ energía digestible; $\mathrm{UFC}=$ unidades forrajeras para caballos; $M N D C=$ materias nitrogenadas digestibles para caballos. 


\section{MARTÍNEZMARÍN}

la Fundación Española para el Desarrollo de la Nutrición Animal (FEDNA, 2003). Para estimar el valor nutricional de los alimentos (tabla I) se utilizaron las ecuaciones apropiadas del conjunto de ellas utilizadas en los sistemas americano (Fonnesbeck, 1981; NRC, 1989) y francés (Martin-Rosset et al., 1984; Martin-Rosset et al., 1994; Vermorely Martin-Rosset, 1997; Martin-Rosset, 2001; INRA, 2002; Martin-Rosset et al., 2006).

\section{FORMULACIÓN}

Las restricciones aplicadas a las materias primas no forrajeras derivaron de la experiencia personal y de la literatura y tuvieron en cuenta limitaciones tanto fisiológicas como tecnológicas (Revuelta, 1963; Piccioni, 1970; Leroy, 1974; Wolter, 1977; INRA, 1990; Lewis, 1991; Payne et al., 1994; Pérez, 1995). Las restricciones se expresa- ron como el ratio entre el límite de inclusión de cada materia prima en el concentrado y el porcentaje total de concentrado que podría entrar a formar parte de la ración. En el caso de los forrajes, el límite inferior calculado de acuerdo con lo señalado en el apartado Alimentos se expresó como la cantidad mínima de nutriente forraje que debería ser aportado por unidad de energía de la ración. Respecto a las restricciones aplicadas a los nutrientes (tabla II), en el caso de la proteína se tuvo en cuenta que los aportes pueden ser muy superiores a las necesidades sin ocasionar trastornos aparentes en los caballos siempre que no padezcan disfunciones hepáticas o renales (Lewis, 1991) o estén sometidos a ejercicio muy intenso (Meyer, 1987; Miller-Grabber et al., 1991), por ello, el límite superior se consideró un 50\% mayor que el límite inferior (INRA, 1990). Las res-

Tabla II. Restricciones aplicadas a los nutrientes en la formulación. (Restrictions applied to the nutrients in the formulation).

\begin{tabular}{|c|c|c|c|c|c|c|c|c|c|c|}
\hline & \multicolumn{4}{|c|}{ Yeguas } & \multirow{2}{*}{\multicolumn{2}{|c|}{$\begin{array}{c}\text { Potros } \\
\text { en crecimiento }\end{array}$}} & \multirow{2}{*}{\multicolumn{2}{|c|}{$\begin{array}{c}\text { Caballos de } \\
\text { ejercicio }\end{array}$}} \\
\hline & & & \multicolumn{2}{|c|}{ gestantes } & \multicolumn{2}{|c|}{ en lactación } & & & & \\
\hline & & & min. & máx. & min. & máx. & $\min$. & máx. & $\min$. & máx. \\
\hline \multirow[t]{2}{*}{ Energía } & NRC89 & Mcal ED $/ \mathrm{kg} \mathrm{MS}$ & 1,97 & 2,62 & 2,26 & 2,83 & 2,63 & 3,27 & 2,05 & 2,73 \\
\hline & INRA90 & UFC/kg MS & 0,48 & 0,64 & 0,71 & 0,89 & 0,68 & 0,85 & 0,54 & 0,72 \\
\hline \multirow[t]{2}{*}{ Proteína } & NRC89 & g PB/Mcal & 44 & 66 & 50 & 75 & 45 & 68 & 40 & 60 \\
\hline & INRA90 & gMNDC/UFC & 101 & 152 & 107 & 161 & 107 & 161 & 69 & 104 \\
\hline Fibra & NRC89 & & & & & & & & & \\
\hline bruta & e INRA90 & $\%$ & N/D & 28 & N/D & 25 & N/D & 25 & $\mathrm{~N} / \mathrm{D}$ & 28 \\
\hline \multirow[t]{2}{*}{ Almidón } & NRC89 & g/Mcal & N/D & 127 & $\mathrm{~N} / \mathrm{D}$ & 88 & N/D & 77 & $N / D$ & 122 \\
\hline & INRA90 & g/UFC & N/D & 521 & N/D & 281 & $N / D$ & 295 & $\mathrm{~N} / \mathrm{D}$ & 463 \\
\hline \multirow{2}{*}{ Lisina } & NRC89 & g/Mcal & 1,52 & $\mathrm{~N} / \mathrm{D}$ & 1,77 & $\mathrm{~N} / \mathrm{D}$ & 1,89 & $\mathrm{~N} / \mathrm{D}$ & 1,41 & $\mathrm{~N} / \mathrm{D}$ \\
\hline & INRA90 & $\%$ & N/D & $\mathrm{N} / \mathrm{D}$ & N/D & $\mathrm{N} / \mathrm{D}$ & 0,40 & $\mathrm{~N} / \mathrm{D}$ & $\mathrm{N} / \mathrm{D}$ & $\mathrm{N} / \mathrm{D}$ \\
\hline \multirow[t]{2}{*}{ Calcio } & NRC89 & g/Mcal & 1,78 & $6 \times P$ & 2,30 & $6 \times P$ & 1,65 & $3 \times P$ & 1,46 & $6 \times P$ \\
\hline & INRA89 & g/UFC & 7,29 & & 7,30 & & 6,36 & & 5,56 & \\
\hline \multirow[t]{2}{*}{ Fósforo } & NRC89 & g/Mcal & 1,27 & $\mathrm{Ca}: 1,2$ & 1,24 & Ca: 1,2 & 0,90 & $\mathrm{Ca}: 1,2$ & 0,98 & Ca: 1,2 \\
\hline & INRA89 & g/UFC & 5,21 & & 3,93 & & 3,45 & & 3,70 & \\
\hline \multirow[t]{2}{*}{ Sodio } & NRC89 & g/Mcal & 0,71 & 0,82 & 0,49 & 0,57 & 0,47 & 0,54 & 0,68 & 0,79 \\
\hline & INRA89 & g/UFC & 2,92 & 3,36 & 1,57 & 1,81 & 1,82 & 2,10 & 2,59 & 2,98 \\
\hline \multirow[t]{2}{*}{ Forraje } & NRC89 & g/Mcal & 254 & S/L & 177 & $\mathrm{~S} / \mathrm{L}$ & 153 & $S / L$ & 244 & $\mathrm{~S} / \mathrm{L}$ \\
\hline & INRA90 & g/UFC & 1042 & $S / L$ & 562 & $S / L$ & 591 & $S / L$ & 926 & $\mathrm{~S} / \mathrm{L}$ \\
\hline
\end{tabular}

Abreviaturas: $\mathrm{ED}=$ energía digestible; $\mathrm{UFC}=$ unidades forrajeras para caballos; $\mathrm{MNDC}=$ materias nitrogenadas digestibles para caballos; $N / D=$ no disponible; $S / L=$ sin límite.

Archivos de zootecnia vol. 58, núm. 223, p. 336. 


\section{SISTEMAS NRC E INRA Y RACIONES A MÍNIMO COSTE PARA CABALLOS DE OCIO}

tricciones para la fibra bruta y el almidón fueron únicamente límites superiores y los valores se tomaron de las recomendaciones bibliográficas (Wolter, 1977; Tisserand, 1979; Cuddeford, 2001). Los límites para el calcio y el fósforo se establecieron de acuerdo a las necesidades y a la relación entre ellos tal y como se ha señalado en el apartado Necesidades nutritivas. El límite superior absoluto para el calcio se fijó en un valor 5 veces mayor que las necesidades (Jordan et al., 1975). Al sodio se le aplicó de forma tentativa un límite superior equivalente a 1,15 veces el límite inferior calculado de acuerdo a las necesidades. El aporte de vitaminas y oligoelementos se supuso cubierto por la inclusión en el concentrado de una premezcla comercial al 1\%. Los límites mínimo y máximo de la energía se especificaron como unidades por $\mathrm{kg}$ de materia seca $\mathrm{y}$ corresponderían a la concentración necesaria para satisfacer las necesidades cuando el consumo de materia seca fuera máximo y mínimo respectivamente. El límite superior para la fibra bruta se expresó como porcentaje de la ración. La necesidad mínima de lisina para potros según INRA90 es un valor porcentual de la ración y como tal se aplicó. Para el resto de los nutrientes, los límites mínimos y máximos de inclusión se expresaron en función de la concentración energética de la ración, esto es, en g por unidad de energía. Los precios aplicados a las materias primas para la optimización correspondieron a la media de los precios reales de dos fábricas de pienso españolas de diferente ubicación geográfica en el período noviembre 2005 a octubre 2006. Para la optimización de las raciones a coste mínimo se utilizó el programa WinFeed 2.8 (WinFeed, 2006).

\section{ANÁLISIS DELOS RESULTADOS}

Con el programa SPSS 15.0 (SPSS, 2006) se realizó un análisis de la varianza según el modelo:

$$
Y_{i j k}=\mu+\alpha_{i}+\beta_{j}+\gamma_{k}+\alpha \beta_{i j}+\alpha \gamma_{i k}+\beta \gamma_{j k}+\varepsilon_{i j k}
$$

siendo:

$\mathrm{Y}_{\mathrm{ijk}}=$ variable dependiente;

$\mu=$ media general

$\alpha_{i}=$ efecto del estado ( $i=1$ a 4 );

$\beta=$ efecto del forraje ( $j=1$ a 3 );

$\gamma_{k}=$ efecto del sistema ( $k=1$ a 2$)$;

$\alpha \beta_{i j}=$ interacción estado*forraje;

$\alpha \gamma_{\mathrm{ik}}=$ interacción estado*sistema;

$\beta \gamma_{\mathrm{jk}}=$ interacción forraje*sistema;

$\varepsilon_{\mathrm{ijk}}=$ error residual.

\section{RESULTADOSYDISCUSION}

De las 24 soluciones posibles, se obtuvieron 23 soluciones válidas capaces de satisfacer las restricciones de ingredientes y de nutrientes planteadas. La solución imposible correspondió a la ración con heno de alfalfa para caballos de acuerdo a las necesidades nutritivas de INRA90.

\section{CONSUMODIARIO}

El consumo diario calculado de materia seca que proporcionó la solución más económica para cada estado fisiológico, tipo de forraje y sistema se muestra en la tabla III. Dicho consumo expresado en relación al $\mathrm{PV}^{0,75}$ (datos no mostrados) fue diferente entre los cuatro estados fisiológicos $(\mathrm{p}<0,05)$, alcanzando el valor máximo en las yeguas lactantes $\left(11,2 \mathrm{~kg} / 100 \mathrm{~kg} \mathrm{PV}^{0,75} / \mathrm{d}\right) \mathrm{y}$ el mínimo en las yeguas gestantes $(7,9 \mathrm{~kg} /$ $\left.100 \mathrm{~kg} \mathrm{PV}{ }^{0,75} / \mathrm{d}\right)$. También se encontraron diferencias entre forrajes $(p<0,05)$ con el mayor consumo en las raciones con la mezcla paja-heno de alfalfa $\left(9,8 \mathrm{~kg} / 100 \mathrm{~kg} \mathrm{PV}{ }^{0,75} /\right.$ d) y el menor en las raciones con heno de alfalfa $\left(9,0 \mathrm{~kg} / 100 \mathrm{~kg} \mathrm{PV}^{0,75} / \mathrm{d}\right)$. Las raciones de INRA90 tuvieron más consumo $(\mathrm{p}<0,05)$ que las de NRC89 (9,6 vs. 9,4 kg/100 kg $\left.\mathrm{PV}^{0,75} / \mathrm{d}\right)$. La interacción estado* sistema fue significativa $(p<0,05)$ con una diferencia mínima entre sistemas en los potros $(0,13$ $\mathrm{kg} / 100 \mathrm{~kg} \mathrm{PV} 0,75 / \mathrm{d}$ ) y máxima en las yeguas lactantes y los caballos donde los consumos calculados para las raciones de INRA90 fueron, respectivamente, un $7,4 \%(1,07 \mathrm{~kg} /$ $100 \mathrm{~kg} \mathrm{PV} 0,75 / \mathrm{d})$ y un $4,9 \%(0,4 \mathrm{~kg} / 100 \mathrm{~kg}$ $\mathrm{PV}^{0,75 / d)}$ superiores a las de NRC89. En las 
MARTÍNEZMARÍN

Tabla III. Consumo calculado de materia seca y nutrientes y coste diario de las raciones optimizadas. (Calculated intake of dry matter and nutrients and daily cost of the optimized rations).

Materia seca Coste Mcal UFC PB MNDC ALM Lis Ca $P$ Na ExPB

CT CF CC $\in / d \quad$ ED/d $/ d \quad$ g/d $\quad$ g/d $\quad$ g/d $\quad$ g/d $\quad$ g/d $\quad$ g/d $\quad$ g/d $\quad$ g/d

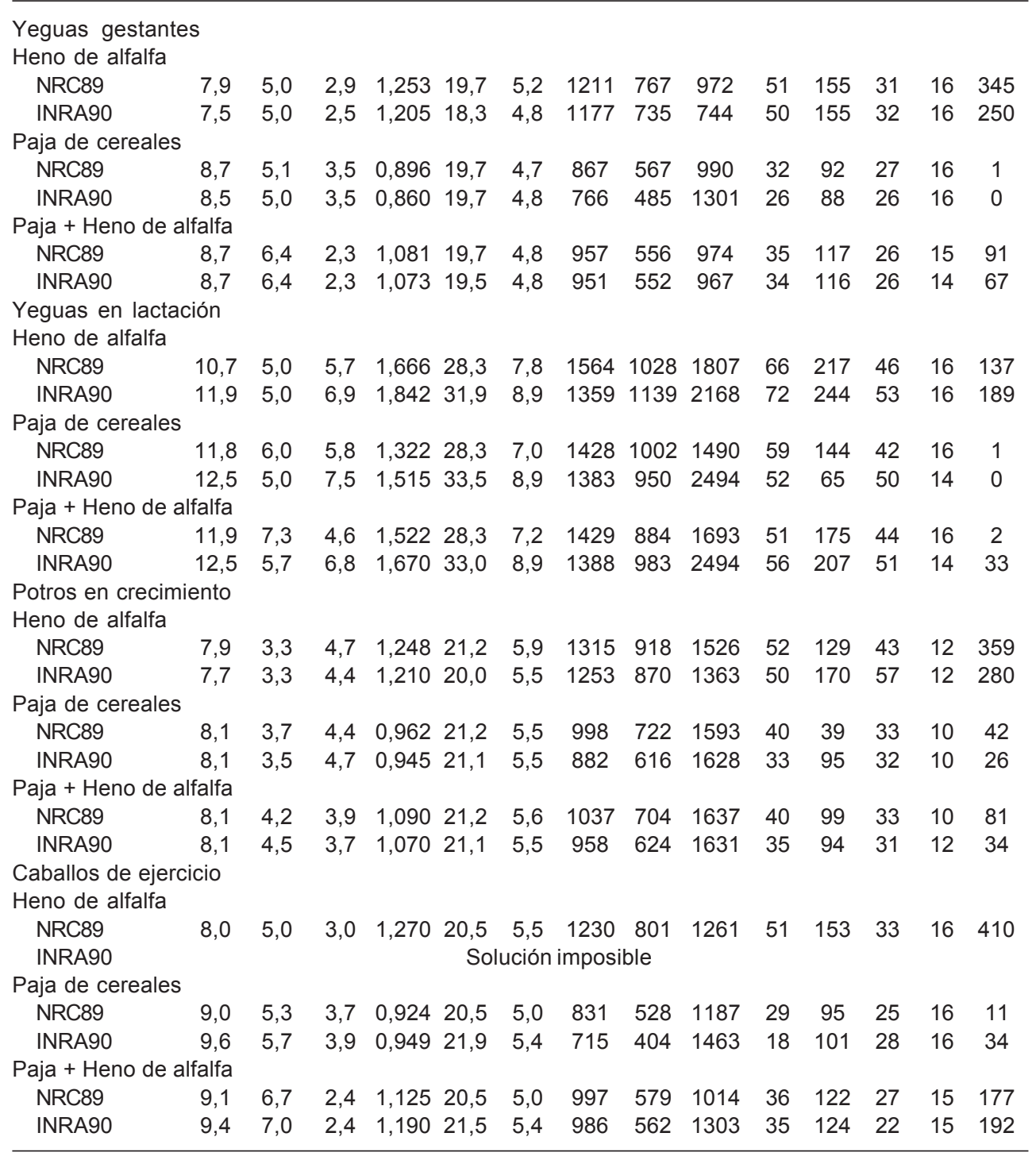

$C T=$ consumo total $\mathrm{kg} / \mathrm{d} ; \mathrm{CF}=$ consumo forraje $\mathrm{kg} / \mathrm{d} ; \mathrm{CC}=$ consumo concentrado $\mathrm{kg} / \mathrm{d} ; \mathrm{ExPB}=$ exceso de proteína bruta; $\mathrm{ED}=$ energía digestible; $\mathrm{UFC}=$ unidades forrajeras para caballos; $\mathrm{PB}=$ proteína bruta; $\mathrm{MNDC}=$ materias nitrogenadas digestibles para caballos; $\mathrm{ALM}=$ almidón; Lis= lisina.

Archivos de zootecnia vol. 58, núm. 223, p. 338. 


\section{SISTEMAS NRC E INRA Y RACIONES A MÍNIMO COSTE PARA CABALLOS DE OCIO}

publicaciones más antiguas (Leroy, 1974; Wolter, 1977) se recomendaba respetar unas relaciones definidas entre el aporte de materia seca y de energía (coeficiente de volumen) a la hora de componer las raciones de los caballos con objeto de no sobrepasar la capacidad del tubo digestivo. De las relaciones señaladas por Wolter (1977) se derivan consumos medios de materia seca que caen dentro de los rangos acotados en el presente trabajo: 9,1 $\mathrm{kg} / \mathrm{d}$ para yeguas gestantes, $11 \mathrm{~kg} / \mathrm{d}$ para yeguas lactantes, $6,3 \mathrm{~kg} / \mathrm{d}$ para potros y $9,9 \mathrm{~kg} / \mathrm{d}$ para caballos Las raciones obtenidas fueron optimizadas en base a dos repartos diarios, lo que es adecuado desde el punto de vista nutritivo de acuerdo a Gill y Lawrence (1998) quienes no encontraron mejoras en la digestibilidad de los nutrientes al aumentar el número de repartos de 2 a 16 comidas/d.

\section{CONSUMOdEFORRAJE}

La proporción de forraje en las diferentes raciones osciló entre 39,9\% (ración de yeguas lactantes con paja de cereales de INRA90) y 74,8\% (ración de caballos con la mezcla paja-heno de alfalfa de INRA90). Ninguno de los consumos calculados de forraje (tabla III) fue superior a los valores estimados como máximos que se pueden derivar de los trabajos de Dulphy et al. (1997a), Dulphy et al. (1997b) y St. Lawrence et al. (2001). El consumo expresado en relación al $\mathrm{PV}^{0,75}$ (datos no mostrados) tendió a ser diferente entre los cuatro estados fisiológicos $(p=0,070)$ y también se detectó una tendencia a la interacción estado* sistema $(p=0,095)$, de forma que la diferencia entre ambos sistemas fue mínima en los potros y las yeguas gestantes $(0,03$ y $0,07 \mathrm{~kg} / 100 \mathrm{~kg}$ $\left.\mathrm{PV}^{0,75} / \mathrm{d}\right)$, sin embargo, fue $0,8 \mathrm{~kg} / 100 \mathrm{~kg}$ $\mathrm{PV}^{0,75} / \mathrm{d}$ menor y $0,5 \mathrm{~kg} / 100 \mathrm{~kg} \mathrm{PV}^{0,75} / \mathrm{d} \mathrm{ma-}$ yor en las yeguas lactantes y en los caballos, respectivamente, con las raciones de INRA90. Comparando entre forrajes, el mayor consumo $(\mathrm{p}<0,05)$ correspondió a la mezcla paja-heno de alfalfa $(6,1 \mathrm{~kg} / 100 \mathrm{~kg}$ $\left.\mathrm{PV}^{0,75} / \mathrm{d}\right)$ y el menor al heno de alfalfa $(4,7 \mathrm{~kg} /$ $\left.100 \mathrm{~kg} \mathrm{PV}^{0,75} / \mathrm{d}\right)$. El efecto del sistema no fue significativo $(\mathrm{p}=0,555)$.

\section{CONSUMOdECONCENTRADO}

El mayor consumo calculado de concentrado (tabla III) correspondió a las yeguas lactantes $(6,2 \mathrm{~kg} / \mathrm{d})$ y el menor a las yeguas gestantes $(2,8 \mathrm{~kg} / \mathrm{d})$. El consumo expresado en relación al $\mathrm{PV}^{0,75}$ (datos no mostrados) difirió entre estados, forrajes y sistemas $(\mathrm{p}<0,05)$, existiendo además una interacción estado*sistema $(\mathrm{p}<0,05)$. Así, el consumo fue mínimo en las raciones de yeguas gestantes y de caballos $(2,7$ y $2,9 \mathrm{~kg} / 100 \mathrm{~kg}$ $\mathrm{PV}^{0,75} / \mathrm{d}$ ) y máximo en las de yeguas lactantes y potros $\left(5,9\right.$ y $\left.5,6 \mathrm{~kg} / 100 \mathrm{~kg} \mathrm{PV}^{0,75} / \mathrm{d}\right)$. Las raciones con paja de cereales aportaron más concentrado $\left(4,8 \mathrm{~kg} / 100 \mathrm{~kg} \mathrm{PV} V^{0,75} / \mathrm{d}\right)$ en comparación con las de heno de alfalfa $(4,5 \mathrm{~kg} /$ $100 \mathrm{~kg} \mathrm{PV} 0,75 / \mathrm{d}$ ) y, sobre todo, con las de la mezcla paja-heno de alfalfa $(3,7 \mathrm{~kg} / 100 \mathrm{~kg}$ $\left.\mathrm{PV}^{0,75} / \mathrm{d}\right)$. En conjunto, las raciones de INRA90 supusieron un consumo de concentrado superior en $0,3 \mathrm{~kg} / 100 \mathrm{~kg} \mathrm{PV}^{0,75} / \mathrm{d}$, ya que, aunque hubo similitudes en las raciones de las yeguas gestantes, los potros y los caballos (diferencia de 0,14; 0,10 y $0,08 \mathrm{~kg} / 100 \mathrm{~kg} \mathrm{PV} V^{0,75} / \mathrm{d}$ ), las raciones para las yeguas lactantes superaron en $1,6 \mathrm{~kg}$ / $100 \mathrm{~kg} \mathrm{PV}^{0,75} / \mathrm{d}$ a las de NRC89. Wolter et al. (1971) concluyeron que la alimentación de caballos de ejercicio con concentrados granulados en cantidades comprendidas entre 8 y $12,5 \mathrm{~kg} / \mathrm{d}(1,5 \%$ a $2,4 \% \mathrm{PV})$ en función del contenido en fibra bruta (de 2,9 a $13,7 \%$ ) y con acceso exclusivamente a paja de cereales de la cama es satisfactoria desde el punto de vista metabólico y fisiológico. Con las raciones del presente trabajo, el consumo de concentrado osciló de 0,95 a $1,5 \% \mathrm{PV}$ con valores de fibra bruta comprendidos entre 6 y $10,9 \%$ (datos no mostrados).

\section{ENERGÍA}

Como era de esperar hubo diferencias $(p<0,05)$ en el consumo calculado de ED y UFC entre los distintos estados. También fueron significativas $(p<0,05)$ las diferen- 
cias entre forrajes y sistemas y las interacciones estado*sistema y forraje*sistema. El consumo calculado de ED y de UFC (tabla III) fue mayor en las raciones de INRA90 frente a las raciones de NRC89 (1,07 Mcal/ d y $0,38 \mathrm{UFC} / \mathrm{d})$. Las raciones para las yeguas lactantes de INRA90 tuvieron de media un $15,9 \%$ más de ED y un $21,9 \%$ más de UFC que las de NRC89. En el resto de estados, las diferencias entre ambos sistemas fueron inferiores al 3,8\% tanto para la ED como para las UFC. La diferencia en el consumo de energía entre los dos sistemas en función del tipo de forraje fue máxima en las raciones con paja ( $1,62 \mathrm{Mcal} / \mathrm{d}$ y $0,6 \mathrm{UFC} / \mathrm{d})$ y mínima en las raciones con la mezcla pajaheno de alfalfa $(0,23 \mathrm{Mcal} / \mathrm{d}$ y $0,05 \mathrm{UFC} / \mathrm{d})$. Estos resultados indican una estimación muy distinta del contenido energético de la paja y una diferente valoración de las necesidades energéticas, especialmente en las yeguas lactantes, entre los sistemas aunque es difícil apuntar cual de los dos es más acertado. Hansson (1934) en base a pruebas con más de 100 raciones expresó las necesidades nutritivas de los caballos como energía neta-Unidades Alimenticias (UA), donde $1 \mathrm{UA}=2100 \mathrm{Kcal}$ EN de lactación-, siendo las necesidades de mantenimiento de $0,9 \mathrm{UA} / 100 \mathrm{~kg}$ PV y el valor energético de la leche de yegua de 0,24 UA/kg (Leroy, 1974), por tanto, las necesidades totales de las yeguas lactantes del presente estudio serían de 8,1 UA o 7,5 UFC (1 UA=0,93 UFC). Según esto, la EN necesaria para yeguas lactantes calculada según Hansson es un $15,7 \%$ inferior a las recomendaciones de INRA90 (7,5 vs. 8,9 UFC/d). Por otro lado, las necesidades de ED para las yeguas lactantes de NRC89 y del sistema alemán (Coenen, 2001) solamente se diferencian en un $4,6 \%$ (28,3 vs. 29,6 Mcal/d).

\section{ProteínA}

Los consumos calculados de PB y MNDC (tabla III) fueron diferentes $(p<0,05)$ por efecto del estado, forraje y sistema de alimentación. El consumo fue mayor en las yeguas lactantes y los potros (1425 y 998 y 1073 y $742 \mathrm{~g} / \mathrm{d}$ ), en las raciones con heno de alfalfa $(1280$ y $880 \mathrm{~g} / \mathrm{d} v s .1089$ y 681 y 984 y $659 \mathrm{~g} / \mathrm{d}$ en las raciones con paja-heno de alfalfa y con paja de cereales) aunque con diferencias entre estados (estado*forraje $\mathrm{p}<0,05)$, y en las raciones de NRC ( $7 \%$ más de PB y 4\% más de MNDC). En cuanto al exceso de aporte de proteína (tabla III) sobre las necesidades mínimas establecidas, no hubo diferencias $(p=0,295)$ entre los sistemas. Sin embargo, sí fue significativo $(\mathrm{p}<0,05)$ el efecto del estado, del forraje y la interacción estado*forraje. El consumo calculado de proteína en las raciones con heno de alfalfa fue de media un $17 \%$ superior a los requerimientos mínimos establecidos incluso en los estados fisiológicos de mayor demanda nutricional como son la lactación o el crecimiento. Según estos resultados, la inclusión de heno de alfalfa como único forraje en las raciones de caballos, independientemente de su estado fisiológico, dificulta la aproximación de los aportes nitrogenados a las necesidades diarias. La excreción renal de la urea resultante del metabolismo hepático del exceso de proteína provoca un mayor consumo de agua y la acumulación de amoníaco en el ambiente del establo (Pagan, 1998). No obstante, en ponis se ha comprobado que puede existir un reciclaje diario de nitrógeno ureico en intestino grueso de hasta $574 \mathrm{mg} / \mathrm{kg} \mathrm{PV}^{0,75} / \mathrm{d}$ (Prior et al., 1974). Si las circunstancias son similares en caballos, el consumo diario de proteína en exceso que podría ser reciclado en el intestino de un caballo de $500 \mathrm{~kg}$ equivaldría a unos $380 \mathrm{~g}$ de proteína bruta. Dentro de las raciones optimizadas en el presente trabajo, esa cantidad solamente se superó $(410 \mathrm{~g} / \mathrm{d})$ en la ración con heno de alfalfa para caballos de NRC89 y quedó muy próxima en la ración con heno de alfalfa para yeguas gestantes basada en dicho sistema $(345 \mathrm{~g} / \mathrm{d})$.

\section{ALMIDÓN}

El consumo calculado de almidón (tabla 


\section{SISTEMAS NRC E INRA Y RACIONES A MÍNIMO COSTE PARA CABALLOS DE OCIO}

III) fue mayor $(\mathrm{p}<0,05)$ en los estados de mayores necesidades energéticas (2024 y $1563 \mathrm{~g} / \mathrm{d}$ en yeguas lactantes y potros vs. 991 y $1234 \mathrm{~g} / \mathrm{d}$ en yeguas gestantes y caballos) y tendió a ser mayor $(\mathrm{p}=0,09)$ en las raciones con paja de cereales $(3,7 \%$ y $10,2 \%$ más que en las raciones con paja-heno de alfalfa y con heno de alfalfa). De media, las raciones de INRA90 contuvieron un $16 \%$ más de almidón $(216 \mathrm{~g} / \mathrm{d})$ que las de NRC 89 $(p<0,05)$. Las interacciones estado* sistema $\mathrm{y}$ forraje* sistema fueron significativas $(\mathrm{p}<0,05)$ de forma que, respecto a NRC89, el consumo de almidón en las raciones de INRA90 fue un 43,4\% y un 30,9\% superior en las yeguas lactantes y en las raciones con paja de cereales respectivamente. Las diferencias observadas podrían justificarse por la menor valoración energética de la paja de cereales en INRA90, lo que implica una mayor inclusión de materias primas ricas en energía (básicamente cereales) en la ración para poder cubrir las necesidades energéticas especialmente en los grupos con mayores requerimientos. Medina et al. (2002) observaron que en raciones donde el aporte de almidón es próximo a la máxima capacidad digestiva en intestino delgado $(4 \mathrm{~g} / \mathrm{kg}$ $\mathrm{PV} /$ comida), la inclusión de suficiente fibra neutro detergente (FND) para alcanzar una relación FND/almidón= 1 fue capaz de reducir las modificaciones de la flora microbiana, el pH y la concentración de ácido láctico en el intestino grueso normalmente observadas en semejantes circunstancias (Julliand et al., 2001). En este sentido, la relación FND/almidón en las raciones del presente trabajo osciló de 2,2 a 4,9 (datos no mostrados). Además el cereal incluido en el concentrado estaría procesado doblemente (molido fino y calentado con vapor de agua) para conseguir un gránulo de elevada durabilidad, lo que repercutiría favorablemente en la digestibilidad del almidón (Meyer et al., 1995).

\section{LISINA}

El consumo calculado de lisina se mues- tra en la tabla III. Las diferencias por estado, tipo de forraje y sistema, y la interacción forraje* sistema y estado* forraje fueron significativas $(\mathrm{p}<0,05)$. El mayor consumo correspondió a las yeguas lactantes (59vs. 42, 38 y $36 \mathrm{~g} / \mathrm{d}$ en los potros, yeguas gestantes y caballos), a las raciones con heno de alfalfa (56 vs. 40 y $36 \mathrm{~g} / \mathrm{d}$ en las de paja-heno de alfalfa y paja de cereales) y a las raciones de NRC89 (2,6 g/d más que las de INRA90). La diferencia entre el heno de alfalfa y la paja de cereales fue mayor en los caballos y las yeguas gestantes $(26,8$ y $21,5 \mathrm{~g} / \mathrm{d})$ y menor en los potros y las yeguas lactantes $(14,5 \mathrm{y}$ $13,5 \mathrm{~g} / \mathrm{d})$. La diferencia entre sistemas fue más apreciable en las raciones con paja donde el consumo de lisina de las raciones de NRC89 fue 7,8 g/d superior a las de INRA90. Lo más destacable de estos resultados es que, de acuerdo a los mínimos establecidos en la formulación, las raciones de NRC89 aportarían cantidades de lisina iguales o superiores a las necesidades establecidas de acuerdo a las recomendaciones de dicho sistema. Por el contrario, las raciones de INRA90 para yeguas gestantes y caballos con paja de cereales, que no incluyeron mínimos en la formulación, fueron deficientes en lisina $(-4 \mathrm{y}-11 \mathrm{~g} / \mathrm{d})$ según las recomendaciones de NRC89 y, aunque se estableció un porcentaje mínimo de lisina en las raciones de los potros según las recomendaciones de INRA90, el consumo diario fue también inferior a las recomendaciones de NRC89 para dichos animales en las raciones con paja-heno de alfalfa y con paja de cereales $(-5 \mathrm{y}-7 \mathrm{~g} / \mathrm{d})$. En el presente trabajo no se estimó el contenido de otros aminoácidos distintos a la lisina en las raciones obtenidas. En potros se ha comprobado (Graham et al., 1994; Staniar et al., 2001) que la complementación de la ración con treonina una vez cubiertas las necesidades de lisina (relación treonina/lisina de $0,7 / 1$ a 0,8 / 1) resulta en un mayor crecimiento.

COSTEDIARIO DELARACIÓN

El coste de las raciones en euros por 


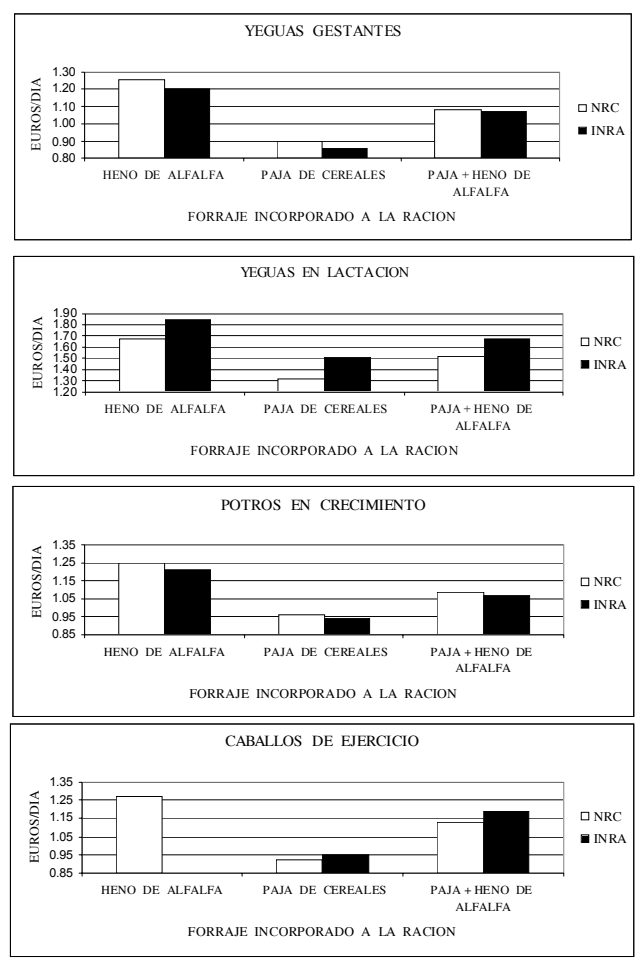

Figura 1. Coste diario calculado de las raciones en función del estado productivo, forraje utilizado y sistema de valoración. (Calculated daily cost of the rations according to the physiological state, forage included in the ration and feed evaluation system).

animal y día está recogido en la tabla III y se muestra gráficamente en la figura 1. Hubo diferencias significativas en el coste $(p<0,05)$ debidas al estado fisiológico, al forraje y al sistema. Entre estados, las raciones más caras fueron las de yeguas lactantes (1,590 euros/d) y las más baratas las de yeguas gestantes $(1,061$ euros/d). Las raciones con paja de cereales fueron más económicas, con una diferencia media de $0,327(31,2 \%)$ y $0,181(17,3 \%)$ euros/d respecto a las raciones con heno de alfalfa y la mezcla paja-heno de alfalfa, respectivamente, aunque este efecto se relacionó con el estado (interacción estado*forraje: $p=$
0,073). Las raciones de INRA90 fueron más caras que las de NRC89 (0,039 euros/d). La interacción estado* sistema fue significativa $(\mathrm{p}<0,05)$ observándose que las raciones para las yeguas gestantes y los potros fueron más económicas cuando se calcularon en base a INRA90 (1,046 y 1,075 vs. 1,077 y 1,100 euros/d). Por el contrario, las raciones para las yeguas lactantes y los caballos fueron más baratas en NRC89 (1,503 y 1,106 vs. 1,676 y 1,147 euros/d). El menor coste de las raciones con paja de cereales indica que, en las condiciones del presente estudio, este forraje es una alternativa válida a otros de mayor calidad nutricional como el heno de alfalfa. Por otro lado, la diferencia de 0,173 euros/d (11,5\%) entre NRC89 e INRA90 en el coste de las raciones de yeguas lactantes estuvo en línea con las diferencias en la inclusión de forraje y de concentrado y en el contenido de energía y almidón de las raciones de ambos sistemas para este grupo de animales.

\section{CONCLUSIONES}

En las condiciones del presente trabajo, las raciones para caballos basadas en forrajes secos y concentrados granulados optimizadas de acuerdo a las necesidades nutritivas y la valoración de alimentos de NRC89 e INRA90 son diferentes en el consumo calculado total, de forraje y de concentrado, y en el coste, aunque el efecto es variable según el estado. Las diferencias en el contenido de energía y almidón son debidas fundamentalmente a la mayores necesidades de energía de las yeguas lactantes y al menor contenido energético de la paja de cereales según INRA90 frente a NRC89. Las diferencias en el aporte de proteína pueden deberse a que INRA90 no indica unas necesidades mínimas de lisina. El hecho de que la utilización de heno de alfalfa como único forraje suponga un derroche de proteína, incluso en los estados fisiológicos con mayores necesidades nitrogenadas, unido a la ventaja económica de la incorporación de 


\section{SISTEMAS NRC E INRA Y RACIONES A MÍNIMO COSTE PARA CABALLOS DE OCIO}

paja de cereales debe ser tenido en consideración en el diseño de raciones para caballos estabulados alimentados a pesebre.

\section{AGRADECIMIENTOS}

A los doctores Pérez Alba y Pérez Hernández por su consejos y revisión críti-

\section{BIBLIOGRAFÍA}

Coenen, M. 2001. German feedings standards. In: Advances in equine nutrition II (ed. Joe D. Pagan and J. Geor). Nottingham University Press. Thrumpton. p. 365-378.

Cuddeford, D. 2001. Starch digestion in the horse. In: Advances in equine nutrition II (ed. Joe D. Pagan and J. Geor). Nottingham University Press. Thrumpton. p. 95-103.

Dulphy, J.P., W. Martin-Rosset, H. Dubroeucq, J.M. Ballet, A. Detour and M. Jailler. 1997a. Compared feeding patterns in ad libitum intake of dry forages by horses and sheep. Factors of variation and prediction. Liv. Prod. Sci., 52: 4956.

Dulphy, J.P., W. Martin-Rosset, H. Dubroeucq and M. Jailler. 1997b. Evaluation of voluntary intake of forage through -fed to light horses. Comparison with sheep. Factors of variation and prediction. Liv. Prod. Sci., 52: 97-104.

FEDNA. 2003. Tablas de composición y valor nutritivo de alimentos para la fabricación de piensos compuestos. 2a edición. (ed. C. De Blas. G.G. Mateos y P.Ga . Rebollar). Fundación Española para el Desarrollo de la Nutrición Animal. Madrid.

Fonnesbeck, P.V. 1981. Estimating digestible energy and TDN for horses with chemica analysis of feeds. J. Anim. Sci., 53: 241-242.

Gill, A.M. and L.M. Lawrence. 1998. Effect of feeding frequency on digestion in ponies. In: Advances in equine nutrition (ed. Joe D. Pagan). Nottingham University Press. Thrumpton. p. 8587

Graham, P.M., E.A. Ott, J.H. Brendemuhl and S.H. TenBroeck. 1994. The effect of supplementa lysine and threonine on growth and development of yearling horses. J. Anim. Sci., 72: 380-386.

Hansson, N. 1934. Alimentación de los animales domésticos. Imp. Juan Pueyo. Madrid. ca. Al doctor Muñoz Serrano por sus orientaciones en el estudio estadístico. A J.M. Sánchez Martín de Piensos Compuestos Coavic y P.J. Ortega Losa de Piensos Vigor por permitir el empleo de los precios de las materias primas de sus empresas. A V. Deike de Novus Spain por la ayuda prestada en la documentación bibliográfica.

INRA. 1990. L'Alimentation des chevaux (ed. W Martin-Rosset). Editorial INRA. Paris.

INRA. 2002. Tables de composition et de valeur nutritive des matières premières destinées aux animaux d'élevage (ed. D. Sauvant, J.M. Perez et G. Tran). Editorial INRA. Paris.

Jordan, R.M., V.S. Meyers, B. Yoho and F.A. Spurrell. 1975. Effect of calcium and phosphorus levels on growth, reproduction, and bone development of ponies. J. Anim. Sci., 40: 78-85.

Julliand, V., A. De Fombelle, C. Drogoul and E. Jacotot. 2001. Feeding and microbial disorders in horses: 1-Effects of an abrupt incorporation of two levels of barley in a hay diet on microbial profile and activities. J. Equine Vet. Sci., 21: 543-546.

Leroy, A.M. 1974. Cría racional del ganado. Ediciones Gea. Barcelona.

Lewis, D.L. 1991. Alimentación y cuidado del caballo. Editorial Intermédica. Buenos Aires.

Martin-Rosset, W. 2001. Feeding standards for energy and protein for horses in France. In: Advances in equine nutrition II (ed. Joe D. Pagan and J. Geor). Nottingham University Press. Thrumpton. p. 245-303.

Martin-Rosset, W., J. Andrieu, M. Vermorel et J.P. Dulphy. 1984. Valeur nutritive des aliments pour le cheval. Dans: Le Cheval. Reproduction, sélection, alimentation, exploitation (ed. R. Jarrige et W. Martin-Rosset). Editorial INRA. Paris. p. 209-238.

Martin-Rosset, W., J. Andrieu, M. Vermorel and M. Jestin. 2006. Routine methods for predicting the net energy and protein values of concentrates for horses in the UFC and MADC systems. Liv. Prod. Sci., 100: 53-69.

Martin-Rosset, W., M. Vermorel, M. Doreau, J.L. Tisserand and J. Andrieu. 1994. The French horse feed evaluation systems and recommen- 


\section{MARTÍNEZMARÍN}

ded allowances for energy and protein. Liv. Prod. Sci., 40: 37-56.

McCutcheon, L.J. 2001. Macrominerals - sodium, potassium and chloride. In: Advances in equine nutrition II (ed. Joe D. Pagan and J. Geor). Nottingham University Press. Thrumpton. p. 339351.

Medina, B., I.D. Girard, E. Jacotot and V. Julliand. 2002. Effect of a preparation of Saccharomyces cerevisiae on microbial profiles and fermentation patterns in the large intestine of horses fed a high fiber or a high starch diet. J. Anim. Sci., 80: 2600-2609.

Meyer, H. 1987. Nutrition of the equine athlete. http://www.centaure-metrix.com/iceep/pdf/ iceep2/_1129110000_001.pdf. Consultado: 08/ 05/07.

Meyer, H., S. Radicke, E. Kienzle, S. Wilke, D. Kleffken and M. Illenseer. 1995. Investigations on preileal digestion of starch from grain, potato and manioc in horses. J. Vet. Med. A., 42: 371381.

Miller-Grabber, P., L. Lawrence, J. Foreman, K. Bump, M. Fisher and E. Kurcz. 1991. Effect of dietary protein level on nitrogen metabolites in exercised quarter horses. http:// www.centaure-metrix.com/iceep/pdf/iceep3/ 1130110442 001.pdf. Consultado: 08/05/07.

NRC. 1989. Nutrient requirements of horses (Fith Revised Edition). National Academy Press. Washington DC.

Pagan, J.D. 1998. Protein requirements and digestibility: a review. In: Advances in equine nutrition (ed. Joe D. Pagan). Nottingham University Press. Thrumpton. p. 43-56.

Payne, J.D., W. Rattink, T. Smith and T. Winowiski. 1994. The pelleting handbook. A guide for production staff in the compound feed industry. Borregaard Lignotech. Sarpsborg.

Pérez, L.M. 1995. Límites superiores e inferiores (restricciones) de ingredientes para caballos. En: Nutrición y alimentación de equinos. Pub.
Master de Equinotecnia. Facultad de Veterinaria. Universidad de Córdoba.

Piccioni, M. 1970. Diccionario de alimentación animal. Editorial Acribia. Zaragoza.

Prior, R.L., H.F. Hintz, J.E. Lowe and W.J. Viser. 1974. Urea recycling and metabolism of ponies. J. Anim. Sci., 38: 565-571.

Revuelta, L. 1963. Bromatología zootécnica y alimentación Animal. $2^{\mathrm{a}}$ edición. Editorial Salvat. Barcelona.

SPSS. 2006. SPSS Base 15.0 User's Guide. SPSS Inc., Chicago.

St. Lawrence, A., R.J. Coleman and L.M. Lawrence. 2001. Relationship between NDF and hay intake in horses: a review of published studies. In: Advances in equine nutrition II (ed. Joe D. Pagan and J. Geor). Nottingham University Press. Thrumpton. p. 117.

Staniar, W.B., D.S. Kronfeld, J.A. Wilson, L.A. Lawrence, W.L. Cooper and P.A. Harris. 2001. Growth of thoroughbreds fed a low-protein supplement fortified with lysine and threonine. J. Anim. Sci., 79: 2143-2151.

Tisserand, J.L. 1979. L'Alimentation pratique du cheval. Editorial A. Leson. Paris.

Vermorel, M. and W. Martin-Rosset. 1997. Concepts, scientific bases, structure and validation of the French horse net energy system (UFC). Liv. Prod. Sci., 47: 261-275.

WinFeed. 2006. WinFeed 2.8 Help Documentation. WinFeed (UK) Limited. Cambridge.

Wolter, R. 1977. Alimentación del caballo. Editorial Acribia. Zaragoza.

Wolter, R., R. Moraillon et B. Toulat. 1971. Aliments complets pour chevaux: noveaux essais. Rec. Méd. Vét., 147: 565-570.

Zeyner, A., C. Geibler and A. Dittrich. 2004. Effects of hay intake and feeding sequence on variables in faeces and faecal water (dry matter, $\mathrm{pH}$ value, organic acids, ammonia, buffering capacity) of horses. J. Anim. Physiol. An. N., 88: 7-19.

Archivos de zootecnia vol. 58, núm. 223, p. 344. 